\title{
Neoliberalización experimental y \\ redes filantrópicas en educación
}

\author{
Saura, Geo \\ Universidad de Granada, Granada, España \\ geosaura@ugr.es
}

\section{Resumen}

El objetivo principal de la comunicación es analizar nuevos procesos de «neoliberalización experimental» en el contexto de la gobernanza política filantrópica en educación, para defender los análisis de la movilidad de las políticas, en lugar de las transferencias políticas, en el campo de la educación comparada. Defiendo la sustitución del término «neoliberalismo» por «neoliberalización», para comprender este fenómeno como un «régimen mutativo». Realizo una revisión de la literatura para sistematizar los resultados más relevantes de las principales investigaciones sobre las políticas rápidas, la movilidad de las políticas y los nuevos procesos de gobernanza global de las redes filantrópicas como proyecto metodológico, en vez de las transferencias políticas. Analizo la neoliberalización como cambios resituados, readaptados y fundamentados por la «experimentación regulatoria» propios del paradigma de las «políticas rápidas», que producen mutaciones geográficas. El siguiente apartado detalla los aspectos metodológicos de la investigación desarrollada en el contexto de la política educativa española como «estudio de caso distendido». El artículo utiliza la etnografía en red mediante la combinación de técnicas de las redes políticas con procesos etnográficos.

El apartado de los resultados muestra y analiza tres tipologías de redes filantrópicas y sus discursos docentes. La «red política global resituada» es la mutación de la red Teach for All en España, Empieza por Educar, que promueve una política rápida, orientada a crear nuevos docentes bajo lógicas discursivas basadas en la performatividad, la efectividad y la rendición de cuentas. La «red política filantrópica conservadora» se configura mediante una fundación, la iglesia católica y la monarquía junto a alianzas empresariales que han actuado en procesos de formación de 50.000 docentes y en más 3.000 escuelas. La «red política filantrópica experimental» es una alianza filantrópica emergente entre fundaciones de empresas importantes españolas y actores simbólicos, orientada a paliar, mediante programas experimentales, los problemas del sistema educativo.

\section{Abstract}

The main aim of this paper is to analyze new processes of "experimental neoliberalization" in the context of philanthropic governance in education to defend incorporating of policy mobilities rather than transfer policy in comparative education field. I defend the substitution of the term "neoliberalism" with "neoliberalization" so as to understand this phenomenon as a "mutative regime". A review of the literature was carried out in order to provide a systematic overview of the most relevant results of the research on fast policies, policy mobilities and the new governance deriving from philanthropic networks as methodological project, rather than policy transfers. I analyze the concept of neoliberalization characterized by changes which are recontextualized, readapted and founded by the "regulatory experimentation" characteristic of the paradigm of "fast policies" produced by geographical mutations. The following section details methodological aspects of the research conducted within the context of Spanish educational policy as a "distended case- study". The paper uses network ethnography by combining the techniques of political networks with ethnographic processes.

The results section details and analyzes three types of philanthropic networks and their teaching discourses. The "resituated political global network" is the mutation of Teach for All network in Spain, "Empieza por Educar", which promotes a fast policy aimed at creating new teachers under discursive logic based on principles such as performativity, effectiveness and accountability. The "conservative political philanthropic network" is comprised of a foundation, the Catholic Church and the monarchy along with business alliances that have taken part in training processes for 50,000 teachers in over 3,000 schools. And the "experimental philanthropic policy network" is an emerging philanthropic alliance between foundations belonging to the largest Spanish companies and symbolic actors, aimed at alleviating problems in the education system through experimental programmes.

Palabras clave: neoliberalización, educación comparada, movilidad de las políticas, filantropía, privatización, docentes.

Keywords: neoliberalization, comparative education, policy mobility, philanthropy, privatization, teachers. 


\section{INTRODUCCIÓN}

Ante el presente contexto de gubernamentalidad neoliberal puesto en marcha tras la última crisis económica global (2007-2009), nos encontramos ante la necesidad de reconsiderar los análisis políticos. Un replanteamiento que deviene del auge de un nuevo régimen de movilidad de las políticas rápidas (Peck, 2011; McCann \& Ward, 2014) que manifiesta que las políticas están siendo creadas y puestas en marcha a través de una gobernabilidad reflexiva, transnacionalizaciones aceleradas de políticas y prácticas, y una mayor movilidad de las técnicas políticas y los creadores de las políticas (Peck \& Theodore, 2015). Ahora las políticas son reformuladas bajo modelos rápidos que se mueven de unos terrenos a otros mediante relaciones de poder, principios ideológicos, comunidades epistémicas, gobernanzas multidireccionales y discursos experimentales que hacen mutar a las políticas en sus viajes (Peck \& Theodore, 2010). Estas formas de movilizar las políticas rápidas, obliga a prestar la atención a los «múltiples espacios superpuestos» (McCann \& Ward, 2012) de la formulación de políticas, donde incluir de forma interconectada la escala, el territorio, el lugar, lo local e incluso lo global junto a redes políticas que verifican que las políticas son movilizadas bajo «ensamblajes globales» (Collier \& Ong, 2005) y tienden a readaptarse bajo una vivacidad continuada de modificación de los regímenes políticos situados.

Aunque todas estas perspectivas críticas de análisis político tienen relación con los intereses e interrogantes que han ocupado al campo de estudio que Dolowitz y Marsh (1996) definieron como "transferencias políticas», es evidente que existen diferencias al respecto. La literatura más ortodoxa sobre las transferencias políticas en educación ha centrado el interés en analizar los procesos de innovación y las resituaciones reformistas desde un contexto a otro dentro de los límites jurisdiccionales en los que se aplican las políticas de cambio. Mayoritariamente desde enfoques racionalistas y descriptivos, ha sido común analizar cómo las políticas se resitúan en espacios delimitados por el Estado-nación mediante procesos de externalización (Schriewer \& Martinez, 2004) o a través de cauces analíticos transferenciales bajo lo que Gita Steiner-Khamsi (2004) sostiene como «policy borrowing and lending». Esta corriente ha estudiado, mediante el uso de los métodos de la comparación de contextos (Steiner-Khamsi, 2004; Steiner-Khamsi \& Waldow, 2012), los actores políticos y sistemas educativos que proponen y reciben los modelos, así como las lógicas sociales y discursivas para la legitimar las adopciones políticas. A través de influencias dirigidas por actores relevantes externos, se han utilizado los conocimientos sobre modelos educativos, acuerdos de gobierno e ideas políticas (Phillips \& Ochs, 2003) que se trasladan para reformular instituciones y prácticas de otros entornos políticos (Dolowitz \& Marsh, 1996). Los estudios sobre las transferencias han partido desde una lógica binaria de lo global y lo local fundamentada en comprender cómo en los procesos de globalización han sido organismos internacionales, instituciones financieras, policy-makers y think-tanks, entre otros, los múltiples actores políticos que han propulsado arquetipos transferenciales hacia sistemas educativos que tenían que ser reformulados. Estos actores, desde diferentes argumentaciones teóricas, metodológicas y contextuales, han impulsado las traslaciones de modelos educativos y prácticas concretas para que los contextos de recepción adecúen sus sistemas educativos a los principios de la globalización (Rappeleye, 2012). Así, la perspectiva sobre las transferencias ha analizado cómo las políticas son trasladadas por procesos de atracción, resonancia y recepciones contextualizadas desde un marco nacional hacia otros mediante procesos transnacionales (Steiner-Khamsi, 2004). También ha habido otros aportes relevantes, previos a la movilidad de las políticas rápidas, interesados en estudiar cómo los discursos se mueven entre el espacio global, el Estado y las instituciones modificando los significados discursivos y prácticos por las interacciones entre esos tres contextos antes de que las políticas sean recontextualizadas o puestas en acto (Beech, 2009; Cowen 2009). Los diferentes estudios de las transferencias políticas se han centrado en comprender cómo hay políticas que se trasladan a espacios específicos bajo principios de elecciones y adopciones políticas que son diseñadas y puestas en marcha por actores políticos concretos que dirigen y legitiman las políticas reformistas. Es un campo de análisis, dentro de la comparación, que se puede resumir en comprender y analizar cuáles son los contextos innovadores y exitosos donde la política funciona, quiénes son los actores que producen la política y cómo se transfieren hacia contextos en los que hay creadores, consumidores y emuladores de la política. 


\section{GOBERNANZA ESTRATÉGICA RELACIONAL DE LA NEOLIBERALIZACIÓN MÓVIL Y EXPERIMENTAL}

En estos momentos, debido a los cambios vertiginosos en las reformulaciones políticas generadas bajo nuevos procesos de neoliberalización que son contextualizados y diferenciados de unos escenarios a otros, el uso del enfoque de la «movilidad de las políticas rápidas» se está situando como una visión más adaptativa que la de las transferencias políticas. Por la necesidad de analizar estos procesos de neoliberalización emerge una nueva generación de investigaciones que está más atenta en prestar atención al contexto socioespacial de las actividades de formulación de las políticas y a las mutaciones híbridas entre las técnicas y las prácticas políticas (Peck \& Theodore, 2015).

Una primera característica del régimen político contemporáneo es que las políticas se mueven en «ensamblajes globales» (Collier \& Ong, 2005) bajo interacciones multidireccionales en las que se configuran las políticas y los regímenes políticos situados de forma viva mediante codificaciones y territorializaciones a la vez que descodifican y desterritorializan los espacios (Deleuze \& Guattari, 1987). Para el análisis de la política educativa bajo estos principios, hay que partir de la idea de que el «conocimiento político circula globalmente» con intereses específicos e influencias que se mueven en «relaciones en red» (Ball, 2012) como parte de los nuevos "regímenes de conocimiento» (Campbell \& Pedersen, 2008). Las políticas no emergen de un lugar concreto y luego se mueven íntegras y sin cambios a través del espacio (McCann \& Ward, 2012), sino que son movilizadas de un sitio a otro reestructurando los espacios de modo fracturado y sintetizado, es decir, en partes o modos adaptativos. Estos cambios en la reformulación de las políticas nos obligan a prestar atención a los procesos de «espacialización de las relaciones sociales» (Ball, 2012: 25) bajo "nuevos espacios del ciclo político» y «movilidad de los discursos» en los que se genera la neoliberalización educativa (Gulson et al., 2017: 3).

Como consecuencia de lo anterior, un segundo aspecto a que tener en cuenta, es que los ensamblajes globales requieren realizar un giro metodológico fundado en el enfoque socio- espacial de la movilidad de las políticas que nos lleva a traspasar la unidad analítica del Estado- nación como punto de partida (Urry, 2007). Ahora, la comparación de contextos como forma de análisis comparado no tiene la utilidad que tenía en el uso de las transferencias. En la actualidad tenemos que mirar más hacia cómo las políticas y las redes de actores se van diseñando y modificando constantemente a través de movimientos ininterrumpidos (McCann \& Ward, 2012). En los procesos de gubernamentalidad contemporánea hay que comprender al Estado como un «enfoque estratégico-relacional» que actúa en una gobernanza neoliberal de «instituciones, organizaciones e interacciones implicadas en el liderazgo político y en la implementación de decisiones que, en principio, vinculan colectivamente a sus sujetos políticos» (Jessop, 2017: 55). Una gobernanza basada en mecanismos y estrategias de coordinación e interdependencia recíproca compleja entre agentes, organizaciones y sistemas funcionales que operan en redes de poder y toma de decisiones más localizadas.

Un tercer fundamento del régimen de la movilidad es la consideración de los procesos de mutación de las políticas. Para ello, se parte de que la neoliberalización contemporánea no puede ser comprendida como un proceso monolítico (England \& Ward, 2017; Springer, 2010). La reformulación de políticas neoliberales es un movimiento contextualizado, dinámico y cambiante bajo condiciones de mutación y vivacidad caracterizadas por procesos de resituación, readaptación y experimentación de las políticas. El movimiento de mutación de la neoliberalización en educación es un régimen vivo que va mutando de un sitio a otro, a la vez que transforma los espacios públicos, las actuaciones en los servicios públicos, las formas de configurar las políticas y los modos de construir sujetos al servicio de la sociedad de modos experimentales. Estas políticas, aunque se meditan, tienden a ser aceleradas y programadas por actores políticos con intereses muy concretos que actúan experimentando y haciendo mutar a las políticas en sus viajes (Peck \& Theodore, 2015).

La cuarta característica de estos procesos experimentales móviles de la neoliberalización implica la necesidad de analizar las redes políticas en las que se entrelazan múltiples actores para liderar las políticas. Estamos ante nuevas redes políticas de gobernanza programáticas que se constituyen para liderar los cambios políticos contemporáneos. Son redes de gobernanza que actúan cruzando, unificando y conectando las estructuras estatales con la sociedad civil mediante «nuevas alianzas, estrategias y horizontes de acción espacio-temporales» (Jessop, 2017: 103). En estas nuevas oportunidades de cambio existen actores políticos privilegiados por poseer el capital, la capacidad de acción en márgenes de tiempo breves y la posibilidad de actuación en escalas globales (Jessop, 2008). En la nueva gobernanza estratégica relacional de la neoliberalización contemporánea están entrando 
en la arena política estatal una nueva filantropía que hibrida prácticas altruistas con las lógicas del capitalismo, que Bishop y Green (2008) denominaron «filantrocapitalismo». Son reconocidos multimillonarios que a través de fundaciones filantrópicas trasladan las lógicas de sus éxitos empresariales a las políticas públicas mediante discursos basados en la erradicación de las desigualdades sociales. El filantrocapitalismo modifica la lógica de la filantropía tradicional o «filantropía científica» (Bishop \& Green, 2008; Frumkin, 2006), por ser un movimiento que actúa en inversiones más amplias, creando indicadores de inversión, estrechando la relación provisión-inversión, evaluando sus políticas y asesorando los cambios. Este movimiento político, para eliminar las desigualdades sociales fundamenta sus acciones filantrópicas en relaciones de mercado. Tras la crisis económica global de la última década, en la que se han reducido las inversiones sociales, el filantrocapitalismo ha emergido al considerar esta coyuntura como una ventana de oportunidades para incorporar sus éxitos empresariales en las reformulaciones políticas. Tal y como mantiene el enfoque de la movilidad de las políticas rápidas, son actores privilegiados por poseer el capital y tener la capacidad de actuación en márgenes comprimidos de tiempo para influir en espacios amplios. Bajo estas oportunidades de actuación experimentales y rápidas, las redes filantrópicas se conforman como comunidades epistémicas que están liderando la reformulación neoliberal en educación (Ball, 2012; Gulson et al., 2017; Olmedo, 2017; Saura, 2016). Para comprender cómo los nuevos actores filantrópicos están actuando en esta gobernanza neoliberal en educación, esta investigación estudia el contexto español como un caso distendido del régimen de la movilidad de las políticas (Peck \& Theodore, 2015), analizando tres redes políticas que conforman comunidades epistémicas que lideran la reformulación política creando y poniendo en marcha nuevas políticas de formación docente.

\section{METODOLOGÍA}

El proceso metodológico de esta investigación se desarrolla a través de tres actividades interconectadas, adaptando la perspectiva de Howard (2002) al análisis de las redes políticas en educación (Ball, 2012; Olmedo, 2017; Saura, 2016²). La primera actividad se basa en una búsqueda extensiva en internet para analizar las narrativas de fundaciones y empresas que conforman las redes políticas, y las trayectorias profesionales de los miembros en diferentes recursos: webs; blogs institucionales de las redes; vídeos de YouTube; páginas de Twitter, Facebook, blogs, Linkedln e Instagram de las redes y de sus miembros; junto a libros, informes y artículos escritos dentro de las redes. Esta primera actividad se lleva a cabo para conocer quiénes conforman las redes, cómo lo hacen, qué pretensiones de actuación tienen y para seleccionar a los informantes claves del trabajo etnográfico. La segunda fase se basa en explorar las conexiones sociales y el «capital red» (Urry, 2007) generado dentro de las redes identificando nodos y casos relevantes que conforman la interacción entre empresas y actores individuales. Las redes políticas se crean con el software NodeXL (ver imágenes 1, 3 y 4). La tercera actividad que conforma la etnografía en red son procesos propios de carácter más etnográfico: realización de 15 entrevistas en profundidad y notas de campo tomadas tras el acceso a diferentes eventos, reuniones, fundaciones y empresas que constituyen las redes para demostrar la interacción social entre los actores y los mecanismos para configurar las políticas. El proceso etnográfico utiliza un muestreo intencional de informantes claves y prosigue con la técnica «snowball sampling» (Noy, 2008) una vez que los participantes proporcionan datos y facilitan el acceso a otras entrevistas y encuentros. Los datos de las diversas fases de la investigación se sistematizan mediante la codificación axial del programa NVivo11. El siguiente apartado de resultados, de forma sucinta, muestra el análisis de tres tipologías de redes filantrópicas, los nodos que conforman cada una de ellas, sus propósitos de actuación en el sistema educativo español y sus finalidades reformistas sobre la formación docente.

\footnotetext{
${ }^{1}$ El proceso metodológico forma parte de la investigación postdoctoral (2015-2018) que se puede encontrar más detallado en: Saura (2016) y Saura (2016b).
} 


\section{RED POLÍTICA GLOBAL RESITUADA}

La red global Teach for All, conformada en la iniciativa filantrópica del matrimonio Bill y Hillary Clinton, Global Clinton Initiative (2007), es una red política global enraizada localmente que está actuando en cuarenta y ocho países mediante programas de liderazgo educativo en el que forman a jóvenes para que ejerzan como docentes en entornos complejos ${ }^{2}$. Es una red política global que muta en sus viajes para resituarse en cada contexto de llegada. En España, aterriza en el año 2011 bajo el nombre de Empieza por Educar (ExE) para actuar en Madrid, Cataluña y País Vasco. La red española es liderada por Ana Botín -reconocida filántropa global y presidenta del Banco Santander- y junto a compañías privadas, bancos, filántropos individuales, Universidades y gobiernos regionales constituyen la red política filantrópica resituada. Con la pretensión de eliminar las desigualdades del sistema educativo español actúa bajo nuevos procesos de privatización mediante alianzas empresariales, instituciones católicas y desregulación pro-mercado educativo (Saura, 2016). Al igual que con la red global TFA ha proliferado la privatización escolar en otros contextos, en España ha producido nuevas ventajas a la escuela privada-concertada católica ya que los líderes formados en ExE ejercen como docentes en su red de centros y, posteriormente, tras el acuerdo entre ExE y la red de Escuelas Católicas, entran a formar parte de forma preferente en su bolsa de empleo. Estos beneficios son propios del «capital red» que se genera entre los nexos de las redes políticas (Urry, 2007). En la política educativa española utilizan modelos sintetizados y experimentales mediante programas de liderazgo, emprendimiento, eficacia, eficiencia, resiliencia y políticas de rendición de cuentas que responsabilizan al profesorado como garantes para eliminar las desigualdades educativas (Saura, 2016b). Trabajan con empresas y fundaciones que apoyan económicamente a la red mediante la aceleración de proyectos empresariales de alto impacto social y nuevos programas de formación docente con lógicas empresarial, junto a nuevos acuerdos políticos con los gobiernos catalán y vasco donde lideran la experimentación del programa de MIR docente.

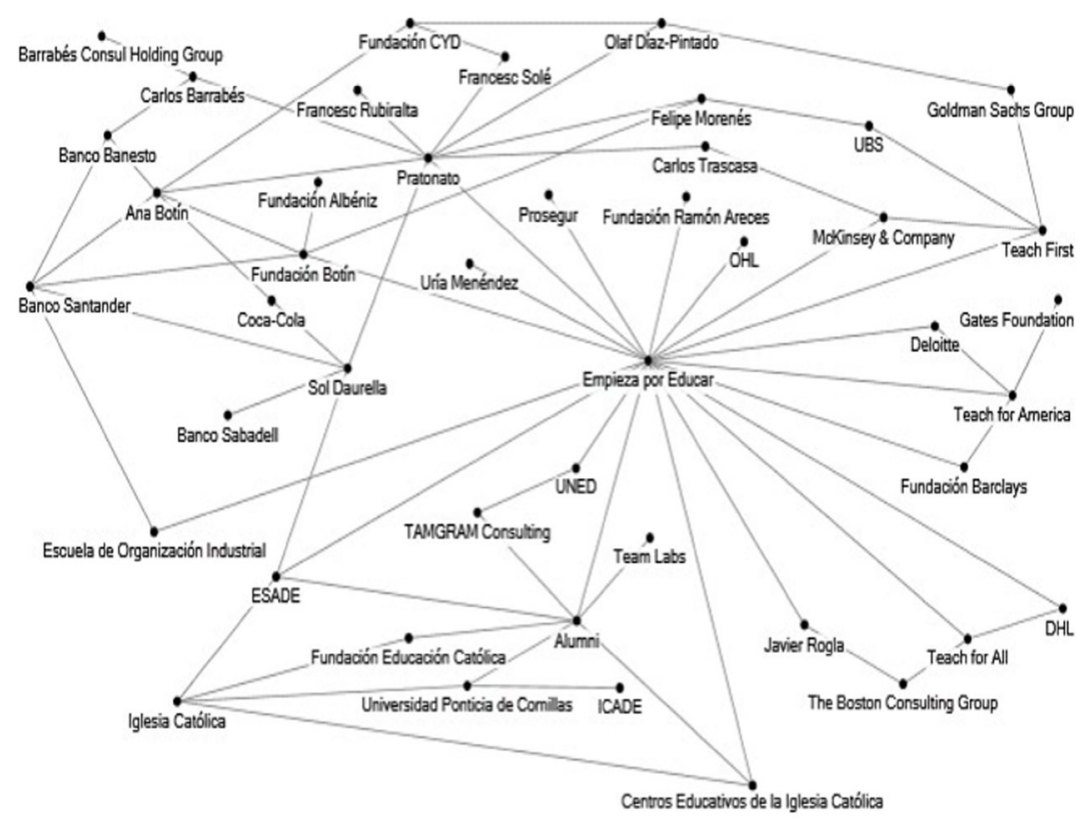

(Imagen 1. Red política filantrópica resituada. Fuente: Saura, 2016b). 


\section{RED POLÍTICA FILANTRÓPICA CONSERVADORA}

Tomando como punto de partida a dos personajes simbólicos de la política educativa española, Carmen Pellicer ${ }^{3}$ y José Antonio Marina ${ }^{4}$, la Iglesia Católica, la monarquía española, gobiernos, universidades privadas, redes empresariales y fundaciones filantrópicas como Ashoka y Fundación Trilema se ha constituido la «red política filantrópica conservadora» (imagen 3 ). Es una nueva comunidad epistémica en el que se entrelaza los diferentes nodos políticos mediante conexiones y contratos económicos para reformar el sistema educativo español. Las políticas que emergen de la red tienen como principales finalidades la incorporación de programas de emprendimiento y la reformulación de procesos de formación docente. La Fundación Trilema ha actuado en procesos de formación de más de 50.000 docentes y 3.000 escuelas. Dirigen la formación docente de las Escuelas Católicas, la gestión y programas formativos de las escuelas Trilema, Másteres Pastorales, elaboración de materiales escolares de las editoriales más relevantes españolas, políticas gubernamentales sobre el emprendimiento empresarial en las escuelas y programas de coaching educativo para la innovación. Bajo las múltiples alianzas generadas en la red, fundamentan sus acciones en programas de formación muy diversos, entre los que destacan: gestión de las aulas, metodologías ágiles, entrenamiento del talento emprendedor, entrenar la competencia espiritual y formación en programaciones y evaluaciones pastorales ${ }^{5}$. A través de discursos sobre "emprendimiento, economía empresarial e innovación educativa» desarrollan sus finalidades transformativas del sistema educativo. Sus discursos sobre coaching educativo y emprendimiento empresarial forman parte de la construcción de racionalidades gubernamentales neoliberales. La gubernamentalidad neoliberal es ejercida mediante racionalidades políticas en la que se conducen las conductas de los sujetos (Foucault, 2004). Su fundamento pedagógico, lo que denominan como «Pedagogías Ágiles del Emprendimiento», lo desarrollan en el programa de formación «Educar el Talento Emprendedor» - avalado por la Fundación Princesa de Girona- para dirigir e innovar la educación con la creación de la «competencia emprendedora» (ver imagen 2).

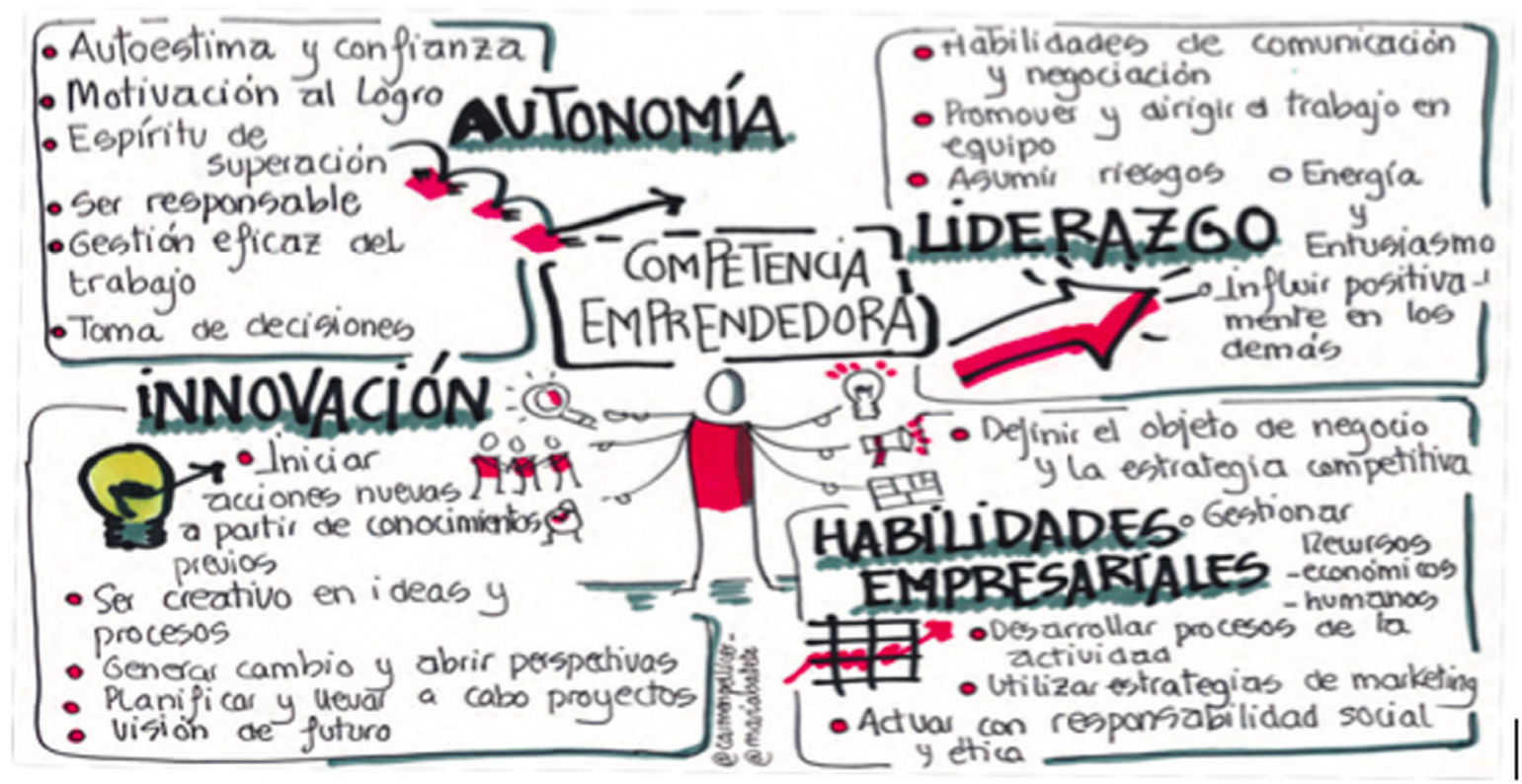

(Imagen 2. Fuente: Pellicer \& Batet, 2017).

\footnotetext{
${ }^{3}$ Presidenta de la Fundación Trilema y de la Asociación Española de Coaching y Evaluación Educativa, Ashoka Fellow 2016 y subdirectora de la Cátedra Nebrija-Santander «Inteligencia Ejecutiva y Educación».

${ }^{4}$ Presidente de la Universidad de Padres y director de la Cátedra Nebrija-Santander «Inteligencia Ejecutiva y Educación».

${ }^{5}$ http://www.fundaciontrilema.org.
} 
La red ha comenzado a movilizar sus políticas en África a través de un programa de formación docente, financiado por la Agencia Española de Cooperación Internacional para el Desarrollo y liderado por Escuelas Católicas, para transformar el sistema educativo de Guinea Ecuatorial mediante políticas experimentales basadas en evaluación de desempeño, clases modelo, formación a docentes y liderazgo directivo.

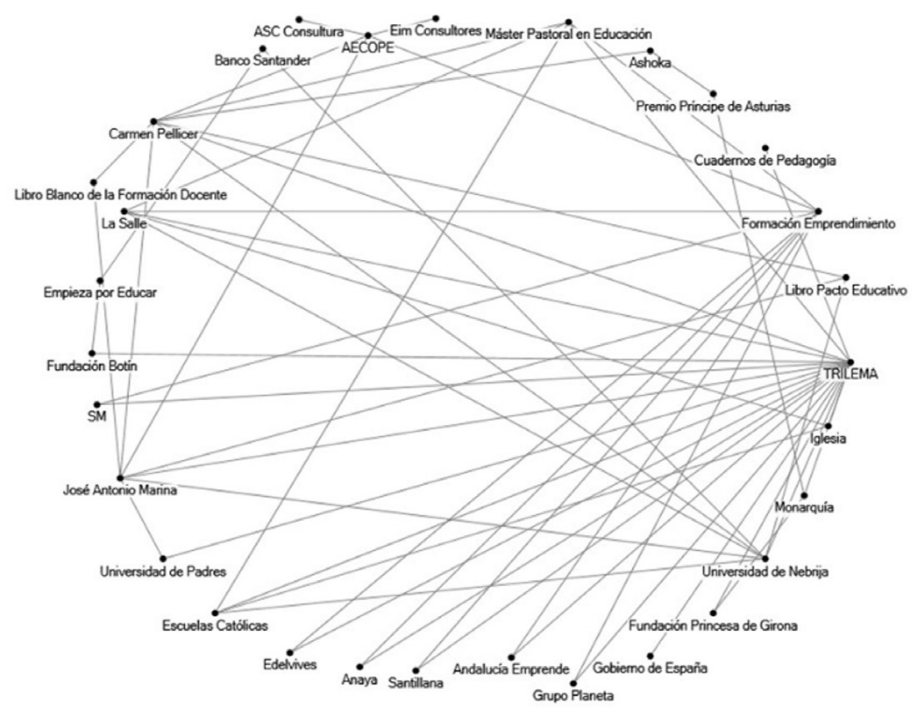

(Imagen 3. Red política filantrópica conservadora).

\section{RED POLÍTICA FILANTRÓPICA EXPERIMENTAL}

Mediante una alianza filantrópica emergente entre fundaciones de empresas del IBEX 35 (Telefónica y CaixaBank), actores simbólicos, fundaciones y gobiernos se constituye la red política filantrópica experimental (ver imagen 4) que tiene como meta paliar las problemáticas del sistema educativo creando políticas experimentales basadas en la innovación en las aulas. En Cataluña, a través de «Escola Nova21», una hibridación público-privada (Fundación Jaume Bofill, Centro UNESCO de Cataluña, Diputación de Barcelona, Universitat Oberta de Catalunya y Fundación EduCaixa) lideran la agenda política para la transformación del sistema educativo mediante la innovación educativa. Etiquetan colegios innovadores y no innovadores que consecuentemente comienzan a producir nuevas lógicas de mercado escolar. Fundamentan su formación en lógicas discursivas de políticas educativas experimentales junto a decisiones programáticas basadas en los estudios de las corrientes «qué funciona» (what works) y «políticas basadas en la evidencia» (evidence-based policy making). Estas lógicas discursivas son propias del nuevo régimen de políticas rápidas (Jessop, 2014; Peck \& Theodore, 2015).

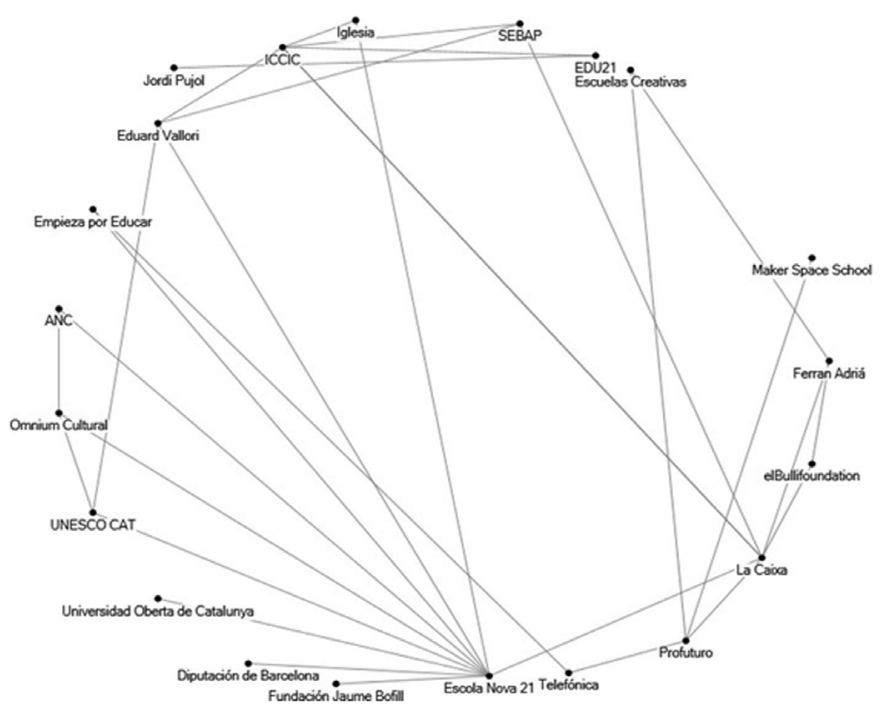

(Imagen 4. Red política filantrópica experimental). 
Dentro de esta red, el programa «Entre Profes» liderado entre la Fundación Telefónica y ExE intenta innovar en la formación docente por medio elementos eminentemente prácticos, en el que graban la docencia para compartir experiencias y crear nuevas formas de control docente. Entre la alianza de la Fundación Telefónica y el «Bullifoundation» (fundación del cocinero Ferran Adrià financiada por CaixaBank) el Proyecto «Escuelas Creativas» tiene como objetivo reformar el sistema educativo innovando y experimentando con la incorporación de los principios de la metodología «Sapiens» por primera vez en educación. Ferran Adrià creó la metodología Sapiens para innovar en la cocina y es considerada un emblema de la experimentación que se ha trasladado mayoritariamente al ámbito empresarial. A través de discursos como: "píldoras educativas», "genoma educativo» y "menú de aprendizaje», el programa de Adrià fundamenta la experimentación constante en educación. Consideran el aula como un laboratorio en el que crear «entornos para fluir, experimentar y crear sin límites» (Barroso, et al., 2017: 54). Un medio para "convertirte en un docente inspirador» y la utilización de la metodología «Design Thinking» bajo "soluciones sencillas, de bajo coste» son otras de sus lógicas sobre la experimentación docente (Aguirre, et al., 2017: 25). Se visibiliza cómo en las reformas móviles rápidas hay redes expansivas generadas por comunidades epistémicas formadas entre gurús, actores de influencia, tecnócratas, evaluaciones de la evidencia, expertos, blogs, vendedores de buenas prácticas, organismos internacionales y compañías empresariales que lideran la reformulación de las políticas experimentales en la docencia.

\section{DISCUSIÓN Y CONCLUSIONES}

En el contexto contemporáneo de las reformulaciones políticas por medio de ensamblajes globales (Collier \& Ong, 2005), el enfoque de la movilidad de las políticas (McCann \& Ward, 2014; Peck \& Theodore, 2015) está siendo más útil que la corriente de las transferencias políticas (Dolowitz \& Marsh, 1996). Este nuevo enfoque sostiene que las políticas son experimentadas, movilizadas y mutadas por diferentes contextos mediante redes de poder, comunidades epistémicas, nuevas formas de gobernanza y modelos sintetizados rápidos (Peck \& Theodore, 2010). En lugar de los métodos y análisis propios de las transferencias políticas en educación comparada (Steiner- Khamsi, 2004) el enfoque de la movilidad de las políticas es una visión más acorde para analizar los procesos de neoliberalización en educación (Gulson et al., 2017). Las políticas educativas se configuran bajo procesos de lo que Jessop (2014) comprende como una «gobernanza estratégica- relacional» en la que múltiples actores constituyen nuevas redes de poder que diseñan y ponen en marcha políticas experimentales y rápidas que mutan en diferentes espacios. En estos procesos de gobernanza rápidos, móviles y experimentales, el filantrocapitalismo (actores políticos que hibridan filantropía con el capitalismo) está ocupando especial relevancia en la arena política educativa configurando y poniendo en marcha nuevas políticas de formación docente. En el contexto de la política educativa española hay tres redes políticas filantrópicas que son re- ensambladas de formas diversas y contextualizadas con fines concretos en los que hay producciones de conocimiento y legitimaciones de las políticas (McCann \& Ward, 2012). Están ocupando especial protagonismo en la configuración de nuevas políticas que inciden en la transformación del sistema educativo mediante los principios de la neoliberalización educativa. La «red política global resituada» es la movilización política de TFA en España por medio de ExE. Es una red constituida entre empresas, bancos, filántropos individuales, universidades y gobiernos con lógicas discursivas que tienen como meta erradicar las desigualdades educativas a través de programas de liderazgo en la formación docente que está beneficiando a los centros católicos y experimentando en políticas gubernamentales. La «red política filantrópica conservadora» la constituyen actores simbólicos, la Iglesia Católica, la monarquía española, gobiernos, universidades privadas, redes empresariales y fundaciones filantrópicas reformulando el sistema educativo con programas formación docente regidos por una innovación de políticas de coaching educativo y emprendimiento empresarial. La «red política filantrópica experimental» es una red de actores políticos, gurús, actores simbólicos, empresas, fundaciones filantrópicas y organismos financieros que tiene como meta transformar la innovación educativa con políticas de experimentación en la docencia. 


\section{REFERENCIAS BIBLIOGRÁFICAS}

Aguirre, A., Barroso, L., Cadenato, M., Domenech, M. E., Garro, J. M., Martínez, B., Poyatos, M., and Vidal, I. (2017). Menú de aprendizaje. Madrid: Fundación Telefónica.

Ball, S. J. (2012). Global Education Inc. New policy networks and the neo-liberal imaginary. London and New York: Routledge.

Barroso, L., de Diego, L. F., García, L., Izquierdo, A., Poyatos, M., Tanarro, J., Ramírez, J. A., and Vivas, M. A. (2017). Mi genoma creativo. Madrid: Fundación Telefónica.

Beech, J. (2009). Policy spaces, mobile discourses, and the definition of educated identities. Comparative Education, 45(3), $347-364$.

Bishop, M. \& Green, M. (2008). Philanthrocapitalism. How Giving Can Save the World. London: Bloomsbury Press.

Campbell, J. L. and Pedersen, O. K. (2014). The National Origins of Policy Ideas. Knowledge Regimes in the United States, France, Germany, and Denmark. New Jersey: Princeton University Press.

Collier, S. J. and Ong, A. (Eds.). (2005). Global assemblages: technology, politics, and ethics as anthropological problems. Malden: Blackwell.

Cowen, R. (2009). The transfer, translation and transformation of educational processes: and their shape-shifting? Comparative Education, 45(3), 315-327.

Deleuze, G. and Guattari, F. (1987). A Thousand Plateaus: Capitalism and Schizophrenia. Minneapolis MN: University of Minneapolis.

Dolowitz, D. P. and Marsh, D. (1996). Who Learns What from Whom? A Review of the Policy Transfer Literature. Political Studies, 44(2), 343-57.

England, K. and Ward, K. (2017). Theorizing neoliberalization. In S. Springer, K. Birch and J. McLeavi (Eds.), The Handbook of Neoliberalism (pp. 50-60). New York and London: Routledge. Foucault, M. (2004). Naissance de la biopolitique. Cours au Collège de France (1978-1979). Paris: Seuil/Gallimard.

Frumkin, P. (2006). Strategic giving: the art and science of philanthropy. Chicago, IL: The University of Chicago Press.

Gulson, K. N., Lewis, S., Lingard, B., Lubienski, C., Takayama, K. and Webb, P. T. (2017). Policy mobilities and methodology: a proposition for inventive methods in education policy studies. Critical Studies in Education, (1-18). DOI: 10.1080/17508487.2017.1288150

Howard, P. H. (2002). Network Ethnography and the Hypermedia Organization: New Media, New Organizations, New Methods. New Media Society, 4(4), 550-574.

Jessop, B. (2008). State Power. A Strategic-Relational Approach. Cambridge: Polity Press. Jessop, B. (2017). El Estado. Pasado, presente y futuro. Madrid: Los Libros de la Catarata. McCann, E. and Ward, K. (2012). Assembling urbanism: Following policies and 'studying through' the sites and situations of policy making. Environment and Planning A, 44(1), 42-51.

McCann, E. and Ward, K. (2014). Policy Assemblages, Mobilities and Mutations: Toward a Multidisciplinary Conversation. Political Studies Review, 10(3), 325-332.

Noy, C. (2008). Sampling Knowledge: The Hermeneutics of Snowball Sampling in Qualitative Research. International Journal of Social Research Methodology, 11(4), 327-344.

Olmedo, A. (2017). Something old, not much new, and a lot borrowed: philanthropy, business, and the changing roles of government in global education policy networks. Oxford Review of Education, 43(1), 69-87.

Peck, J. (2011). Geographies of Policy: From Transfer difusion to Mobility-mutation. Progress in Human Geography 35(6), 773-797.

Peck, J. and Theodore, N. (2010). Mobilizing Policy: Models, Methods and Mutations', Geoforum, 41(2), 169-74.

Peck, J., and Theodore, N. (2012). Follow the Policy: A Distended Case Approach. Environment and Planning, 44(1), 21-36.

Peck, J. and Theodore, N. (2015). Fast policy: Experimental statecraft at the thresholds of neoliberalism. Minneapolis: University of Minnesota Press.

Pellicer, C. and Batet, M. (2017). Pedagogías ágiles para el emprendimiento. Barcelona: AulaPlaneta.

Phillips, D. and Ochs, K. (2003). Processes of Policy Borrowing in Education: Some Explanatory and Analytical Devices. Comparative Education 39(4), 451-461.

Rappeleye, J. (2010). Educational Policy Transfer in an Era of Globalization - Theory, History, Comparison. Frankfurt: Peter Lang. Saltman, K. J. (2010). The Gift of Education: public education and venture philanthropy. New York: Palgrave Macmillan.

Saura, G. (2016). Saving the world through neoliberalism: philanthropic policy networks in the context of Spanish education. Critical Studies in Education, (1-18). DOI: 10.1080/17508487.2016.1194302.

Saura, G. (2016b). Neoliberalización filantrópica y nuevas formas de privatización educativa: La red global Teach For All en España. Revista de Sociología de la Educación, 9(2), 248-264. 
Schriewer, J. and Martinez, C. (2004): Constructions of internationality in education. In G. Steiner- Khamsi (Ed.), The global politics of educational borrowing and lending (pp.29-53). New York: Teachers College Press.

Springer, S. (2010). Neoliberalism and geography: Expansions, variegations, formations.

Geography Compass 4(8), 1.025-1.038.

Steiner-Khamsi, G. (Ed.). (2004). The Global Politics of Educational Borrowing and Lending. New York: Teachers College Press.

Steiner-Khamsi, G. and Waldow, F. (Eds.). (2012). Policy Borrowing and Lending. World Yearbook of Education 2012. London and New York: Routledge.

Urry, J. (2007). Mobilities. Cambridge: Polity Press. 